\title{
Post-Myocardial Infarction Ventricular Septal Defect Six Months following Coronary Artery Bypass Grafting
}

\author{
Amy G. Fiedler, MD, Thoralf M. Sundt III, MD, George Tolis Jr, MD \\ Division of Cardiac Surgery, Massachusetts General Hospital, Boston, Massachusetts, USA
}

\section{ABSTRACT}

Mechanical complications following acute myocardial infarction are associated with high mortality. We present the first reported case of a new post myocardial infarction ventricular septal defect (VSD) within six months of coronary artery bypass grafting. The patient underwent successful surgical correction of the VSD with the assistance of mechanical circulatory support (MCS). This case highlights the importance of mechanical circulatory support in the management of cardiogenic shock associated with rare complications of myocardial infarction, even after surgical revascularization.

\section{INTRODUCTION}

Post myocardial infarction ventricular septal defect (VSD) is a rare mechanical complication following acute myocardial infarction with associated high mortality rates. We present the first documented case of a new post myocardial infarction VSD within six months of surgical revascularization. The patient underwent successful surgical repair with the assistance of intra-aortic balloon counter-pulsion (IABP) and was ultimately discharged from the hospital. This is a unique case, as a new VSD within six months of prior surgical correction is a rare complication infrequently encountered by the cardiac surgeon.

\section{CASE REPORT}

Our patient is a 73-year-old man who underwent three vessel coronary artery bypass grafting (CABG) (left internal mammary artery to the left anterior descending artery, saphenous vein graft [SVG] to the obtuse marginal, SVG to the right coronary artery [RCA]) at an outside institution in January of 2016. In June 2016, he re-presented to the outside institution with chest pain radiating to his jaw and was diagnosed with an inferior ST elevation myocardial infarction (MI). He underwent coronary angiography which demonstrated occlusion of his vein graft to the right coronary artery, and drug eluting stent was placed. During the procedure, he was noted to have a large VSD. As a result, IABP was placed for hemodynamic support and he was transferred to our institution for surgical consideration.

Received fanuary 11, 2017; received in revised form fuly 13, 2017; accepted fuly 14, 2017.

Correspondence: Amy Fiedler, MD, Cox 630, 55 Fruit Street, Boston, MA 02114 2696; 617-724-0800; fax: 617-726-5804 (e-mail: afiedler@partners.org).
The patient arrived to our cardiac intensive care unit with evidence of cardiogenic shock with end organ dysfunction manifested by shock liver and acute kidney injury. IABP counter-pulsion was continued, and the patient was initiated on inotropic support and diuretic therapy. His clinical status improved following 72 hours of medical optimization, at which point the decision was made to bring the patient to the operating room for definitive surgical repair.

A midline sternotomy was performed in a reoperative fashion. The left internal mammary artery graft was identified and controlled. The ascending aorta was cannulated and bicaval venous cannulation was obtained for cardiopulmonary bypass. The operation was conducted at 34 degrees Celsius and myocardial protection was achieved utilizing antegrade and retrograde cardioplegia. The heart was separated from the surrounding pericardium to facilitate rotation and exposure. There was a large infero-posterior infarct identified corresponding to his recent inferior MI. The left ventricle was entered along the course of the posterior descending artery through the area of infarct. The posterior septum was necrotic extending upwards toward the annulus of the mitral valve. A posterior wall pseudoaneurysm was also identified just superior to the level of the VSD. The VSD was repaired with the use of the exclusion technique with a large patch anchored to the posterior mitral annulus and extended up towards the apex with a running 3-0 prolene suture line along the healthy appearing left ventricular endocardium. The suture line was then transferred up toward the left ventricular side of the ventriculotomy, thus closing the left ventricle and excluding the septum and the septal defect on the right side. Two 3-0 prolene sutures were then passed through the patch at the level of the posterior mitral annulus and exteriorized on the posterior wall of the left ventricle, where they were tied over a piece of Teflon felt. This provided a solid anchor to the weakest point of our patch, and the Teflon served as the posterior anchoring edge of our ventriculotomy closure. A series of 3-0 prolenes in horizontal mattress fashion were then used to bring together the two edges of the ventriculotomy and supported on either side by strips of Teflon felt. The patient was separated from cardiopulmonary bypass without difficulty, but the echocardiogram demonstrated new severe mitral regurgitation, thought to be secondary to geometric reconfiguration of the left ventricle due to the VSD repair, or possible inadvertent incorporation of a papillary muscle in a stitch due to the proximity of the mitral valve and subvalvular apparatus to the VSD. The patient was placed back on cardiopulmonary bypass and mitral valve replacement was performed in the standard fashion, utilizing a 29 St. Jude Biocor 
valve. The patient was once again separated from cardiopulmonary bypass without complication. Intraoperative TEE showed no residual VSD and a well-seated mitral bioprosthesis without paravalvular leak.

His postoperative course was largely uneventful. He remained in the intensive care unit until postoperative day two, at which point he was transferred to the surgical floor after his IABP and inotropic support were weaned and discontinued. He was discharged to rehabilitation on postoperative day seven in stable condition.

\section{DISCUSSION}

Post myocardial infarction VSD is an uncommon yet devastating complication. Despite advances in revascularization, surgical technique, percutaneous septal closure devices, and mechanical circulatory support (MCS), mortality remains high. In patients managed medically, mortality rates exceed 90\% [Gilotra 2014]. Those patients who undergo operative intervention still have a mortality rate between $19 \%$ and $60 \%$, which represents the highest mortality rate of all cardiac procedures recorded in the STS database [Arnaoutakis 2012]. Aggressive surgical management remains the definitive treatment of choice; however, with the advent of percutaneous closure devices and mechanical circulatory support, additional options are now available, particularly to assist and temporize the hemodynamically unstable patient in hopes to improve operative mortality.

The role for percutaneous closure devices for VSD continues to evolve. Definitive management has been described in patients with post myocardial infarction VSD in highly selected patients with simple defects $<15 \mathrm{~mm}$ in the sub-acute to chronic setting [Shabestari 2015]. Percutaneous devices have also been shown to stabilize patients with shunt reduction prior to operative intervention, which is advantageous in the patient with severe cardiogenic shock and end organ dysfunction.

MCS has also been reported in the literature as a mechanism to stabilize the patient in severe cardiogenic shock with post myocardial infarction VSD while awaiting surgical correction. IABP was the first MCS device described in 1973 by Austen in the management of five patients who suffered VSD following myocardial infarction. They demonstrated that IABP reduced the effective shunt factor via selective augmentation of systemic cardiac output [Gold 1973]. Additionally, IABP reduces left ventricular afterload, improving both the shunt fraction across the VSD and improving coronary perfusion during diastole. More recently, there have been case reports describing the use of either percutaneous ventricular assist devices such as the Impella 5.0 (AbioMed; Danvers, MA, USA) or extracorporeal membrane oxygenation (ECMO) for temporary stabilization, with promising results [LaTorre 2011].

\section{Conclusion}

This case report details a case of post infarct VSD within six months of definitive surgical revascularization, requiring redo sternotomy with VSD repair. We use this case as a platform to highlight the fundamentals of surgical repair, but also the emerging technologies with percutaneous devices and mechanical circulatory support that have shown promise in improving morbidity and mortality in this high-risk patient population. This case is unique in that a post infarct VSD was successfully treated several months after apparently uneventful coronary artery bypass grafting, secondary to saphenous vein graft occlusion. Mechanical complications of myocardial infarction are rare after coronary bypass, most likely due to the development of an extensive collateral blood supply, which is often seen on angiograms years after the initial revascularization procedure. This extensive collateralization may make the interventricular septum and papillary muscles less susceptible to ischemia, therefore reducing the incidence of post CABG VSD.

\section{REFERENCES}

Arnaoutakis G, Zhao Y, George T, Sciortino C, McCarthy P, Conte J. 2012. Surgical repair of ventricular septal defect after myocardial infarction: outcomes from the Society of Thoracic Surgeons National Database. Ann Thorac Surg 94:436-44.

Gilotra N, Stevens G. 2014. Temporary mechanical circulatory support: a review of the options, indications, and outcomes. Clin Med Insights Cardiol 8:75-85.

Gold H, Leinbach R, Sanders C, Buckley M, Mundth E, Austen G. 1973. Intraaortic balloon pumping for ventricular septal defect or mitral regurgitation complicating acute myocardial infarction. Circulation 57:1191-6.

LaTorre M, Centofanti P, Attisani M, Patane F, Rinaldi M. 2011. Posterior ventricular septal defect in presence of cardiogenic shock: early implantation of the impella recover LP 5.0 as a bridge to surgery. Tex Heart Inst J 38:42-9.

Shabestari M, Ghaderi F, Hamedanchi A. 2015. Transcatheter closure of postinfarction ventricular septal defect: A case report and review of the literature. J Cardiovasc Thorac Res 7:75-7. 